\title{
2 Partizipation und betriebliche Beteiligung
}

Als Modus der Beteiligung ist Partizipation ein „Kernelement demokratischer Systeme“ (Alcántara, Bach, Kuhn \& Ullrich, 2016, S. 1) und in den verschiedensten Zusammenhängen anzutreffen. Neben dem politischen System (ebd.) findet sich der Begriff als „Argumentationsfigur" (Pluto, 2007, S. 25) im schulischen Bereich (Palentien \& Hurrelmann, 2003), in der Arbeitswelt (siehe Abschnitt 1.2) und vielen Bereichen mehr (Schnurr, 2011, S. 1069). Auch das „Engagement von Werkstatträten“ (Schlummer, 2015) bzw. deren Beteiligung (Cannizzaro, Hartmann \& Olivia, 2020; Berg, 2020) oder die „Subjektperspektive“ der Werkstattratsmitglieder (Nieß, 2016) werden unter dem Slogan von Partizipation verhandelt.

Der vielfachen Verwendung entsprechend liegen verschiedene Ansätze, Verständnisse, Definitionen und Begründungen von Partizipation vor. Auch eine Verschmelzung oder Gleichsetzung mit den Begriffen der Teilnahme, der Teilhabe (Bartelheimer, Behrisch, Daßler, Dobslaw, Henke \& Schäfers, 2020, S. 1; Selle, 2010, S. 3), der Beteiligung oder der Mitbestimmung (Kotthoff, 1981, S. 28) ist gängig. Doch um was geht es überhaupt, wenn von Partizipation gesprochen wird? Was ist der Inhalt und das Besondere an dem vielfach verwendeten Konstrukt? Bestehen Unterschiede zu dem Teilhabebegriff der Behindertenhilfe oder bietet sich eine synonyme Begriffsverwendung an, wie dies in der englischen Fachsprache gängig ist (Bartelheimer et al., 2020, S. 51)? Um Antworten zu finden und das „Partizipationsphänomen“ greifbarer zu machen, wird in Abschnitt 2.1 eine Begriffsannäherung vorgenommen. Im Anschluss wird der Bezug zur betrieblichen Beteiligung hergestellt (Abschnitt 2.2) und ein Zwischenfazit gezogen (2.3), mit dem eine Arbeitsdefinition von Partizipation festgehalten wird.

\subsection{Partizipation - eine Begriffsannäherung}

Zur Annäherung an Partizipation beginnt Abschnitt 2.1.1 mit einer knappen Skizze auffälliger Elemente der verschiedenen Begriffsverwendungen bzw. -definitionen. Ergänzend dazu wird der dezidierte Entwurf eines subjektiven Partizipationsverständnisses vorgestellt, der aufzeigt, dass eine sehr spezifische Abgrenzung möglich ist. Um demgegenüber die konzeptionell-planerische Komponente und unterschiedliche Partizipationsintensitäten zu akzentuieren, wird die Begriffsannäherung mit der Vorstellung zweier Stufenmodelle fortgeführt (Abschnitt 2.1.2). Darauf aufbauend wird charakterisiert, wie Partizipation im außerschulischen Fachdiskurs zu Behinderungen derzeit verhandelt wird (Abschnitt 2.1.3).

(C) Der/die Autor(en) 2022

V. Schachler, Partizipation durch Werkstatträte, Beiträge

zur Teilhabeforschung, https://doi.org/10.1007/978-3-658-35383-4_2 


\subsubsection{Geteilte Elemente und Vorstellung eines dezidierten Partizipationsverständnisses}

Häufig wird nicht explizit benannt, was genauer gemeint ist, wenn von und über Partizipation gesprochen wird, insbesondere in politischen Dokumenten und Berichten (z. B. aktueller „Kinder- und Jugendbericht“, Bundestags-Drucksache [BT-Drs.] 18/11050; „Teilhabebericht der Bundesregierung über die Lebenslagen von Menschen mit Beeinträchtigungen“, BMAS, 2013). Offenbar wird davon ausgegangen, dass bekannt ist, was Partizipation bezeichnet, und es keiner genauen Erläuterungen bedarf. Zudem taucht der Partizipationsbegriff - wie einleitend bereits erwähnt - in den unterschiedlichsten Verwendungszusammenhängen auf. Trotz dieser Unterschiedlichkeit und der teilweise fehlenden expliziten Begriffsfassungen zeichnen sich einige geteilte Elemente ab. Akzentuieren lässt sich Folgendes:

- Partizipation wird konsensual - auch ohne explizite Begründungen und Begriffsauslegungen - als wichtig erachtet (bspw. von Kardorff, 2014, S. 9; Scheu \& Autrata, 2013, S. 7). Treffend kommt dies etwa in der Spinat-Allegorie zur Bürgerpartizipation von Arnstein (1969) zum Ausdruck: „Citizien participation is a little like eating spinach: no one is against it in principle because it ist good for you“" (S. 216).

- Partizipation kann durch strukturelle Einschränkungen oder Unterstützungen, etwa in zeitlicher oder monetärer Hinsicht, gefördert oder verhindert werden (Schnurr, 2011, S. 1076).

- Die Ausübung von Partizipation ist voraussetzungsvoll. Es braucht u. a. Gelegenheiten, um für Partizipation benötigte Kompetenzen auszubilden und einzuüben (etwa Wansing, 2012, S. 101).

Unter denjenigen Erörterungen, die sich bemühen, Partizipation vor der Verwendung einzugrenzen und umfänglicher zu definieren, lässt sich das erkenntnistheoretisch entwickelte Verständnis von Partizipation nach Scheu und Autrata hervorheben. Dieses Verständnis ist insofern bemerkenswert, als dieses auf einer umfassenden Herleitung beruht und damit eine klare, abgrenzende Begriffsfassung von Partizipation gefunden wird. Im Folgenden wird dieses Verständnis rudimentär skizziert, für eine genauere Erläuterung lässt sich auf die Monografie „Partizipation und Soziale Arbeit“ von Scheu und Autrata (2013) verweisen.

In der ressourcen- und empowermentorientierten Sozialen Arbeit ist Partizipation als Handlungsmaxime der Praxis ein grundlegender Begriff. Anfänglich im Zuge von Sozialplanung verwendet, wird Partizipation in der Sozialen Arbeit seit den 1990er-Jahren verstärkt unter den Stichworten der „Klienten- bzw. Nutzerpartizipation“ (Schnurr, 2011, S. 1069) diskutiert und folglich in immer mehr Bereichen angewandt. Als Konsequenz daraus ist Partizipation zur „Schimäre“ (Scheu \& Autrata, 2013, S. 7) avanciert. Um Utilitarismus-Tendenzen 
zu begegnen, d. h. „Partizipation wird nur so und da gewünscht, wo sie nützlich ist“ (ebd.), begründen Scheu und Autrata ein eigenes Partizipationsverständnis. Dieses ist nicht nur bruchstückhaft, auf ausgewählte Bereiche und Kontexte gerichtet, sondern begreift Partizipation insgesamt als wesentlich für das gesamte Leben (ebd., S. 7 f.). Zunächst arbeiten Scheu und Autrata heraus, wie sich in den Politikwissenschaften und in anderen sozialwissenschaftlichen Diskursen um eine Begriffsfassung von Partizipation bemüht wurde. Diese wurde nach ihrer Auffassung letztlich nicht erreicht, sondern hat im Ergebnis zu fragmenthaften, paradoxen Auffassungen geführt. So auch in der Sozialen Arbeit, in der Partizipation verkürzt als „Beteiligung an den Leistungen“ (ebd., S. 8) ausgegeben wird. Diesen Befund nehmen Scheu und Autrata zum Anlass, phylogenetisch abzuleiten, wie sich Partizipation historisch entwickelt hat und im sozialen Miteinander einzuordnen ist. Damit begründen sie Partizipation als „eine besondere Form des menschlichen Handelns, die sich dadurch auszeichnet, dass sie auf das subjektiv als Ganzes Gesehene Einfluss nimmt“ (ebd., S. 9). Partizipation zeichnet sich in dieser Betrachtung durch vier zentrale Merkmale aus: als „Handlung, Auswahl unter Handlungsalternativen, gesellschaftliche Teilhabe und Einflussnahme“ (ebd., S. 256). Der zentrale Bezugspunkt ist die subjektive Komponente zur Bestimmung des gesellschaftlichen oder sozialen „Ganzen“. Nach Scheu und Autrata kann sich der Gegenstand und die Zielrichtung von Partizipation, wie etwa die Erweiterung von individueller Lebensqualität oder die solidarische Einflussnahme auf größere gesellschaftliche Bezüge, nur aus der Subjektperspektive bestimmen. Folglich sei Partizipation abzugrenzen ,,von einer Verpflichtung, für Ziele von Gruppen oder anderen Menschen einzutreten“ (ebd., S. 272).

Das Anliegen von Scheu und Autrata, Partizipation genauer zu fassen oder zu systematisieren, greifen gleichfalls andere Autor*innen auf. Beispielsweise werden normative (materiale, aktive) Ansätze von formativen (instrumentellen) unterschieden, verfasste von unverfassten Formen etc. (Nieß, 2016, S. 70 ff.; Weisser, 2012, S. 170 ff.). Die Einteilungen und Abgrenzungen bleiben dabei oftmals etwas grobmaschig und die Übergänge fließend. Eine andere Systematisierungsweise besteht darin, Partizipation in Form von Stufenmodellen zu beschreiben. 


\subsubsection{Stufenmodelle zur Charakterisierung von Partizipationsintensitäten}

Zur Abgrenzung unterschiedlicher Partizipationsvorstellungen bestimmen Stufenmodelle Partizipation, indem sie unterschiedliche Reichweiten von Beteiligungsaktivitäten verdeutlichen und Handlungen anhand der damit möglichen (nicht) einflussnehmenden Intensität bewerten. Im Folgenden werden zwei Modelle vorgestellt.

Ein häufiger Bezugspunkt ist das Partizipationsmodell von Arnstein zur Bürgerbeteiligung aus den 1970er-Jahren. Dieses richtet sich im Kern an der Machtteilhabe aus, die bei Entscheidungen besteht bzw. nicht gegeben ist. Für Arnstein (1969) ist Partizipation ,a categorical term for citizen power“ (S. 216), eine bewusste Umverteilung der politischen und ökonomischen Prozesse und eine Strategie, mit der marginalisierte Gruppen an diesen Prozessen und den Vorteilen wohlhabender Gesellschaften einflussnehmend teilnehmen (ebd.). Um inhaltsleere Beteiligungsformen zu erkennen, beschreibt sie ein achtstufiges Modell. Dieses beginnt mit der Manipulation und Therapie, die als Strategien im Kontext von Bürgerbeteiligung unter dem Slogan einer vermeintlichen Partizipation anzutreffen sind (siehe Abbildung 1).

\section{Nonparticipation}

Therapy

Manipulation

\section{Degrees of citizen} power

Citizen control

Delegated power

Partnership

\section{Placation}

Consultation

Informing

Quelle: Eigene Anfertigung auf Grundlage von Arnstein (1969, S. 217).

Abbildung 1: Partizipationsmodell zur Bürgerbeteiligung von Arnstein (1969)

Mit den Strategien sind z. B. Praktiken gemeint, bei denen Bürger*innen nur ausschnittsweise, unter Darstellung falscher oder unvollständiger Tatsachen manipulativ oder mit dem Ziel der Verhaltensänderung beteiligt werden. Auf einer nächsthöheren Stufe ordnet Arnstein oberflächliche bzw. symbolische Beteiligungsformen ein: die der Information, der Konsultation und der Platzierung. Diese sind wichtige Beteiligungselemente, bleiben als Partizipationsverfahren jedoch unvollständig, da mit ihnen keine Verbindlichkeiten einhergehen, bspw. bei öffentlichen Anhörungen als konsultatives Verfahren (ebd., S. 219). Mit der Partnerschaft, der 
delegierten Macht und der Bürgerkontrolle sind demgegenüber Beteiligungsformen möglich, mit denen eine verbindliche Machtabgabe und -teilnahme erfolgt, so etwa bei Prozessen, bei denen eine geteilte Planungs- und Entscheidungsfindung zwischen den Akteuren grundlegend feststeht und diese nicht einseitig verändert werden können. Ab der Stufe der Partnerschaft ordnet Arnstein Partizipation ein. Charakteristisch geht hierbei die Einforderung der Machtteilhabe von Bürger*innen aus. Weitergehend können Verhandlungen darin münden, dass es zu Formen der delegierten Macht kommt. Hier sind es die Bürger*innen, die (behördliche) Prozesse dominant bestimmen. Abschließend ist in diesem Modell die Bürgerkontrolle der höchstwertige Ausdruck von Partizipation. Hierbei obliegen nun alle Entscheidungsbefugnisse den Bürger*innen, ebenso die monetäre Entscheidungsgewalt (ebd., S. 221 ff.).

Arnstein beschreibt ihr Modell als Instrument, das in verschiedenen Handlungsfeldern angewandt werden kann. Dennoch ist sie sich bewusst, dass ihr Modell eine (plakative) Vereinfachung komplexer Zusammenhänge ist und sowohl marginalisierte als auch machthabende Gruppen sehr heterogen sind und keine homogenen Interessen vertreten (ebd., S. 217).

Große Beachtung erfährt gleichfalls das neunstufige Partizipationsmodell aus der Gesundheitsförderung und Prävention von Wright, von Unger und Block (2010), etwa zur Entwicklung partizipativer Forschung (Bergjold, 2013). Dieses Modell ist eine Weiterentwicklung von Arnsteins Ausarbeitung und baut auf der Prämisse von Partizipation als „Teilhabe an Entscheidungsprozessen“ (Wright et al., 2010, S. 19) auf. Als ein Ergebnis aus Forschungsprojekten mit praktischem lebensweltorientiertem Anwendungsbezug steht bei Wright et al. die realisierbare Handlungsebene im Vordergrund und ein Bewusstsein dafür, dass sich Partizipation entwickeln muss. Wie in Abbildung 2 dargestellt, gehen auch sie von einem Modell aus, in dem Partizipation anhand der Einflussnahme an Entscheidungsprozessen graduell gestaffelt wird. Begonnen bei den Vor- oder Entwicklungsstufen der Partizipation (Einbeziehung, Anhörung, Information) über die partizipativen Stufen (Entscheidungsmacht, teilweise Entscheidungskompetenz, Mitbestimmung) bis zur Selbstorganisation sei eine möglichst große Einbeziehung von Zielgruppen anzustreben. Allerdings können nach ihrem Verständnis hohe Stufen nicht per se realisiert werden und diese eine Berechtigung haben, etwa die Anweisung als Form der Nicht-Partizipation ,z. B. im Fall einer akuten Gefahr (Krankheit, Kindesmissbrauch)“ (ebd., S. 43). 
Vorstufen der

Partizipation

Einbeziehung

Anhörung

Information

\section{Selbstorganisation} geht über Partizipation hinaus

\section{Partizipation}

Entscheidungsmacht

Teilweise Ent-

scheidungskompetenz

Mitbestimmung

Nicht-Partizipation: Anweisung, Instrumentalisierung

Quelle: Eigene Anfertigung auf Grundlage von Wright, von Unger und Block (2010, S. 42).

Abbildung 2: Partizipationsmodell nach Wright, von Unger und Block (2010)

Obwohl Wright et al. für ihr Modell eine gestufte Abbildungsweise wählen, betonen sie, dass dies keine unmittelbare Linearität ausdrückt. Kontextspezifisch können bereits niedrige Partizipationsstufen qualitätsvoll sein und Lernprozesse bei allen Beteiligten anregen (ebd., S. $48 \mathrm{f}$.).

Mitunter werden Stufenmodelle von Partizipation recht kritisch betrachtet. Ihnen wird eine mangelnde Komplexität oder Eindimensionalität angekreidet (Nieß, 2016, S. 84). Gleichfalls können die Abstufungen etwas beliebig wirken. Nichtsdestotrotz sind sie mit ihrer Illustrationsprägnanz hilfreiche Mittel zur Differenzierung unterschiedlicher Beteiligungsformen und können unpräzise Thematisierungen versachlichen wie bspw. in dem außerschulischen Diskurs von Behinderung und Teilhabe.

\subsubsection{Partizipation im Diskurs von Behinderungen und Teilhabe}

Während in spezifischen Handlungsfeldern wie der Kinder- und Jugendhilfe (etwa Pluto, 2007) und in der Sozialen Arbeit insgesamt Partizipation ein tradierter Begriff ist, ist die explizite Beachtung von Partizipation in der Behindertenhilfe vergleichsweise jung (siehe hierzu auch Schnurr, 2011 im Vergleich zu Schnurr, 2018). Wenngleich der Impetus von Partizipation in der Behinderten(selbsthilfe)bewegung (Waldschmidt, 2009) und der dort vertretenen Maxime „Nichts über uns ohne uns!“ (Heiden, 2014) schon länger anklingt und sich die Leistungserbringung des SGB IX auf eine $\mathrm{zu}$ erzielende Selbstbestimmung der 
leistungsberechtigten Personen ausrichtet (§ 1 Abs. 1 SGB IX), fokussierte sich der deutschsprachige außerschulische Fachdiskurs zu Behinderungen und Beeinträchtigungen bisher eher auf andere Begrifflichkeiten. Chronologisch lässt sich herausarbeiten, dass der Partizipationsbegriff im größeren Umfang 2001 mit der International Classification of Functioning, Disability and Health (ICF) der Weltgesundheitsorganisation (WHO) über die internationale Ebene in den hiesigen Fachjargon eintrat. In der Klassifikation ist participation ein wesentlicher Begriff, der ein gewandeltes Verständnis von Behinderung zum Ausdruck bringt. Übersetzt wird participation hier mit „Partizipation [Teilhabe]“ als duales Begriffspaar. ${ }^{6}$ Beschrieben wird dieses Begriffspaar als „Einbezogensein einer Person in eine Lebenssituation“, als „gesellschaftliche Perspektive der Funktionsfähigkeit“ (WHO, 2005, S. 146). Eine andere Handhabung findet sich in der deutschsprachigen Übersetzung der UN-BRK, in der participation bzw. to participate mit Teilhabe und Teilnahme übersetzt ist. Auch die sogenannte „Schattenübersetzung“ der UN-BRK, die in Teilen einer anderen Wortwahl folgt, wich an diesen Stellen zunächst nicht von der amtlichen Übersetzung ab (Beauftragte der Bundesregierung für die Belange behinderter Menschen, 2014). Eine explizite Infragestellung dieser Übersetzung ist lediglich vereinzelt auszumachen (etwa Beck, 2013; Heiden, 2014; Hirschberg, 2010; Nieß, 2016). Offen bleibt, ob mit dieser Wortwahl ein Bedeutungsverlust der einflussnehmenden (politischen) Komponente verbunden ist. Vor diesem Hintergrund kann zur Fachdiskussion von Behinderungen und Partizipation Folgendes akzentuiert werden:

- Teilhabe und Partizipation tauchen als Begriffspaar auf.

- In Texten, die einen der zwei Begriffe genauer beschreiben, werden mitunter identische Inhalte thematisiert. So werden sowohl Teilhabe als auch Partizipation als „Zugang zu Handlungsfeldern und Lebensbereichen“ (Beck, 2013, S. 4) und als „Beteiligung in Form von Mitwirkung und Mitbestimmung“ (ebd.) beschrieben (die Zitate beziehen sich auf Partizipation, zur äquivalenten Beschreibung von Teilhabe siehe Aktionsbündnis Teilhabeforschung, 2015, S. 3).

- Die Begriffe können in der hiesigen Fachsprache dennoch nicht synonym verwendet werden: Sie entstammen unterschiedlichen Kontexten, womit Partizipation eine politische/demokratietheoretische und Teilhabe eine sozialpolitische/-rechtliche Prägung zur Sicherung

\footnotetext{
${ }^{6}$ Dies wird wie folgt begründet: „Die Übersetzung des englischen Begriff[s - sic] ,participation’ ist ,Teilhabe'. Da ,Teilhabe' in der Schweiz jedoch eine engere Bedeutung hat als in Deutschland, dieser Begriff in Deutschland jedoch im Sozialrecht eine zentrale Bedeutung hat, ist der englische Originalbegriff mit ,Partizipation [Teilhabe]' wiedergegeben.“(WHO, 2005, S. 4)
} 
von Leistungen bekommt (Beck, 2013, S. 4; de Nève \& Olteanu, 2012, S. 13; Nieß, 2016, S. 69; Wansing, 2012, S. 96).

- Der Diskurs richtet sich auf Teilhabe als Leitbegriff aus, wie dies in den Bezeichnungen „Teilhabebericht“ (BMAS, 2013), „Bundesteilhabegesetz“ und „Teilhabeforschung“ (Bartelheimer et al., 2020) zum Ausdruck kommt.

- Mit dieser Ausrichtung werden eher sozialrechtliche Zugangs-, Teilnahmeprozesse oder weitere Deutungsperspektiven der Lebensführung (Bartelheimer et al., 2020, S. 43 ff.) fokussiert und weniger ein einflussnehmendes, machtvolles Handeln von Menschen mit Behinderungen.

- Es sind eher vereinzelte Arbeiten, die explizit mit der Begrifflichkeit von Partizipation erscheinen oder sich darunter verorten. ${ }^{7}$

Wenngleich Teilhabe derzeit das Leitkonzept des interdisziplinären außerschulischen Fachdiskurses zu Behinderungen ist und Partizipation im Wortlaut der deutschen Übersetzung der UN-BRK nicht genannt ist, gilt Partizipation dennoch als „Querschnittsanliegen“ (Hirschberg, 2010), als „Gebot“ (Heiden, 2014) oder als „Grundsatz“ (BMAS, 2013, S. 28) der UN-BRK. Zudem werden verschiedene Stellen der Konvention als Partizipationsverankerung ausgelegt (Hirschberg, 2010, S. 3), bspw. Artikel 27 UN-BRK Absatz 1 Buchstabe c, der sich auf die gleichberechtigte Ausübung der Arbeitnehmer- und Gewerkschaftsrechte von Menschen mit Behinderungen bezieht, womit $u$. a. die betriebliche Beteiligung der Personengruppe eingefordert wird.

\subsection{Betriebliche Beteiligung}

Traditioneller Bestandteil der Arbeitnehmerrechte ist in Deutschland die betriebliche Mitbestimmung. In der gesellschaftlichen Gestaltung der BRD ist diese ein konstitutives Element

${ }^{7}$ Bspw. greifen Düber, Rohrmann und Windisch (2015) und die Beiträge in ihrem Herausgeberband die Frage auf, wie Partizipation barrierefrei ermöglicht werden kann. Waldschmidt (2009) fragt in ihrem Beitrag „Politische Partizipation von Menschen mit Behinderungen und Benachteiligungen“ wie es um diese in Deutschland bestellt ist. Hierbei beschreibt sie politische Partizipationschancen (etwa in Form der Wahlbeteiligung, des ehrenamtlichen Engagements etc.) und unterscheidet drei behinderungsspezifische kollektive Aktionsformen: die Selbsthilfegruppe, den Behindertenverband und die Behindertenbewegung. Für eine Übertragung des subjektiven Partizipationsverständnisses von Scheu und Autrata auf das Feld der Behindertenhilfe lässt sich auf Nieß (2016), für eine anwendungsbezogene Arbeitshilfe zur Verbesserung der Partizipationsmöglichkeiten in Einrichtungen, Diensten, Kommunen und Gruppen auf den „Index für Partizipation“ (Denninger, Grüber \& Markowski, 2019) verweisen. 
der hiesigen Arbeitsbeziehungen, im soziologischen Terminus industrielle (Austausch-)Beziehungen genannt. ${ }^{8}$ In den Nachkriegsjahren der BRD formte sich für den privat-gewerblichen Sektor der Arbeitsbeziehungen das duale System bestehend aus der Tarifautonomie nach Art. 9 Abs. 3 Grundgesetz (GG) ${ }^{9}$ und der Betriebsverfassung aus, womit die autonome Aushandlung von Tarifverträgen auf überbetrieblicher Ebene sowie die Arbeitsbeziehungen im Betrieb separat geregelt sind. Gleichfalls dual gestaltet ist betriebliche Mitbestimmung auf den Ebenen des Unternehmens und des Betriebes, in Form des Aufsichtsrats und des Betriebsrats (Müller-Jentsch, 2007, S. 46 ff.). In den Ebenen der Wirtschaft und des unmittelbaren Arbeitsplatzes konnte eine weitergehende demokratische Mitbestimmung gesetzlich hingegen nicht eingeführt werden, weswegen das hiesige System als „Koloß auf tönernen Füßen“ (Diefenbacher \& Nutzinger, 1980, S. 105) bezeichnet wird. Damit ist gemeint, dass betriebliche Mitbestimmung in den Bereichen des Wirtschaftslebens sowie des lebensweltlich-direkten Arbeitsumfeldes nicht verankert ist, womit zentrale (gestaltende) Einflusssphären fehlen.

Auf der Ebene des Betriebs ist die Vertretung der Arbeitnehmer*innen mit dem Betriebsverfassungsgesetz (BetrVG) aus dem Jahr 1972 (mit einer zentralen Neufassung 2001) für den privat-gewerblichen Sektor einheitlich geregelt. ${ }^{10}$ Der öffentliche Dienst sowie Tendenzbetriebe und Religionsgemeinschaften sind von der Anwendung explizit ausgenommen ( 118 BetrVG). Im Folgenden werden die rechtlichen Grundlagen der Betriebsratsarbeit knapp skizziert und die Merkmale des deutschen Modells der Arbeitsbeziehungen herausgearbeitet (Abschnitt 2.2.1). Die Vorgaben des BetrVG sind für Werkstatträte insofern bedeutsam, als ihre

\footnotetext{
${ }^{8}$ Diese Bezeichnung rekurriert auf die englische Begriffsverwendung industrial relations. Sie wird verwendet, um die ,wirtschaftlichen Austauschverhältnisse und sozialen Kooperations- und Konfliktbeziehungen zwischen Kapital und Arbeit beziehungsweise den sie repräsentierenden Akteuren“ (Müller-Jentsch, 1999, S. 7) wertneutral zu benennen. Anders als die Bezeichnung industrielle Beziehungen vermuten lässt, bezieht sie sich keineswegs nur auf den industriellen Bereich, sondern umfasst die Gesamtheit der (organisierten) Beziehungen in der Arbeitswelt (ebd., S. 7 f.). In einer weiter gefassten Definition sind industrielle Beziehungen ,jene Netzwerke, Institutionen und Systeme, in die die verschiedenen Akteurinnen und Akteure der Arbeitswelt eingebunden sind und mit denen sie ihre wirtschaftlichen Austauschverhältnisse und sozialen Konfliktbeziehungen faktisch gestalten und normativ regeln" (Verlag Barbara Budrich, $n$. d.). Im Kern bleibt die Bezeichnung industrielle Beziehungen ein soziologischer Fachbegriff, der sich in anderen Disziplinen, die gleichfalls mit betrieblicher Mitbestimmung beschäftigt sind, nicht behaupten konnte. In synonymer Verwendung ist hier die Bezeichnung Arbeitsbeziehungen gängig (Jakobi, 2007, S. 22).

${ }^{9}$ Grundgesetz für die Bundesrepublik Deutschland in der im Bundesgesetzblatt Teil III, Gliederungsnummer 100-1, veröffentlichten bereinigten Fassung, das zuletzt durch Artikel 1 u. 2 Satz 2 des Gesetzes vom 29. September 2020 (BGBl. I S. 2048) geändert worden ist.

${ }^{10}$ Betriebsverfassungsgesetz in der Fassung der Bekanntmachung vom 25. September 2001 (BGB1. I S. 2518), das zuletzt durch Artikel 6 des Gesetzes vom 20. Mai 2020 (BGBl. I S. 1044) geändert worden ist.
} 
Gremienstrukturen in Anlehnung daran entstanden sind. Anschließend wird auf die Mitarbeitervertretungen im kirchlichen Bereich eingegangen (Abschnitt 2.2.2), denen Werkstatträte in ihrer Arbeit begegnen. Anschließend werden demokratietheoretische Bezüge und funktionelle Deutungen zur Betriebsratsarbeit vorgestellt (Abschnitt 2.2.3). Zu guter Letzt wird der Bezug zum Partizipationsbegriff fokussiert und analysiert, inwieweit es sich bei Betriebsräten um ein Partizipationsinstrument handelt (Abschnitt 2.2.4).

\subsubsection{Rechtliche Grundlagen der Betriebsratsarbeit}

Als Interessenvertretung der Arbeitnehmer*innen (ohne leitende Angestellte) kann ein Betriebsrat ab einer Betriebsgröße von fünf wahlberechtigten Personen gewählt werden $(\S 1 \mathrm{Be}-$ trVG). Eine Pflicht zur Einrichtung besteht nicht. Betriebsrat und Arbeitgeber arbeiten ,vertrauensvoll“ (§ 2 Abs. 1 BetrVG), ohne „Maßnahmen des Arbeitskampfes“ (§ 74 Abs. 2 Satz 1 BetrVG) zusammen. Arbeitskämpfe können lediglich über tariffähige Parteien (§ 2 Abs. 1 BetrVG) und Gewerkschaftsmitglieder angeregt werden, wobei sich der Betriebsrat nicht beteiligen darf (Fricke, Grimberg, Havemann \& Wolter 2018, S. 173 f.). Gewerkschaften muss zur Umsetzung des BetrVG ein Betriebszugang gewährt werden (§ 2 Abs. 2 BetrVG), grundsätzlich bleibt der Betriebsrat jedoch gewerkschaftsunabhängig organisiert. Das BetrVG gilt nicht für leitende Angestellte, die im BetrVG mit bestimmten Voraussetzungen wie Prokura oder selbstständigen Personalentscheidungen ( $§ 5$ Abs. 3 BetrVG) charakterisiert sind. Deren Interessenvertretung richtet sich nach dem Gesetz über Sprecherausschüsse der leitenden Angestellten aus. ${ }^{11}$

Für die Geschäftsführung des Betriebsrats ist vorgesehen:

- Wahl einer*s Vorsitzenden (§ 26 BetrVG);

- Gründung eines Betriebsausschusses zur laufenden Geschäftsführung und ggf. weiteren Aufgabenübertragung ab einer Gremiumsgröße von neun Personen ( 27 BetrVG). Bei kleineren Betriebsräten kann die Geschäftsführung durch den Vorsitzenden oder Einzelpersonen übernommen werden ( 27 Abs. 3 BetrVG). Unter laufender Geschäftsführung werden „Aufgaben wie: Erledigung des Schriftverkehrs, Informationen der BR-Mitglieder, Organisation des BR-Büros und ähnliches“ (Fricke et al., 2018, S. 76) verstanden.

\footnotetext{
11 Sprecherausschußgesetz vom 20. Dezember 1988 (BGBl. I S. 2312, 2316), das zuletzt durch Artikel 8 des Gesetzes vom 20. Mai 2020 (BGBl. I S. 1044) geändert worden ist.
} 
Genaueres sollte in einer Geschäftsordnung ( $\$ 36$ BetrVG) festgelegt werden, mit der sich der Betriebsrat strukturieren kann;

- optionale weitere Ausschüsse und Arbeitsgruppen zur Aufgabenübertragung ab einer Betriebsgröße von 101 Arbeitnehmer*innen ( $§ 28$ und 28a BetrVG). Empfohlen wird, die Aufgaben der Ausschüsse „,auf die vorbereitende Bearbeitung von Sachfragen“ (Fricke et al., 2018, S. 79) zu begrenzen, um Entscheidungsbefugnisse des Betriebsrats nicht zu reduzieren;

- nicht-öffentliche Betriebsratssitzungen ( $\S 29-35$ BetrVG) mit schriftlichen Sitzungsniederschriften und der optionalen, beratenden Teilnahme von Gewerkschaften, der Schwerbehindertenvertretung oder geladener Gäste. Beschlussfassungen erfolgen nach dem Mehrheitsprinzip der stimmberechtigten Anwesenden als Regelfall. Ein bestimmter zeitlicher Turnus ist für die Sitzungen nicht vorgesehen. Für den Arbeitgeber besteht nach Einladung eine Teilnahmepflicht. Dringend empfohlen wird, Beschlussfassungen nicht in dessen Anwesenheit durchzuführen. Einen Protokollanspruch hat der Arbeitgeber lediglich für die Tagesordnungspunkte, zu denen er geladen war (Fricke et al., 2018, S. 84 ff.);

- ehrenamtliche Amtsausführung mit Freistellungs- und Schulungsansprüchen ( $§ 37$ und 38 BetrVG). Betriebsratsarbeit findet als bezahlte Arbeit nach dem Grundsatz der ,Selbstfreistellung“" (Fricke et al., 2018, S. 94) statt. Spezielle Genehmigungspflichten bestehen nicht, eine Abmeldung beim direkten Vorgesetzten wird als ausreichend betrachtet. Gleiches gilt für Seminarbesuche: Diese obliegen der betriebsrätlichen Beschlussfassung und werden dem Arbeitgeber lediglich informatorisch mitgeteilt. Für den Schulungsanspruch wird zwischen einem erforderlichen unbegrenzten ( $\$ 37$ Abs. 6 BetrVG) und einem auf drei bzw. vier Wochen ( $\$ 37$ Abs. 7 BetrVG) begrenzten ,geeigneten“ Anspruch unterschieden, die getrennt voneinander bestehen (Fricke et al., 2018, S. 99);

- anberaumte Sprechstunden während der Arbeitszeit (§39 BetrVG). Arbeitnehmende können diese mit einfachen Abmeldungen, ohne besondere Genehmigungs- oder inhaltliche Auskunftspflichten besuchen (Fricke et al., 2018, S. 106).

Die im Rahmen der Betriebsratsarbeit entstehenden Kosten trägt der Arbeitgeber (§ 40 Abs. 1 BetrVG). Über die notwendigen Ausgaben entscheidet der Betriebsrat. Genehmigungen durch den Arbeitgeber sind für diese nicht notwendig (Fricke et al., 2018, S. 107). Die Errichtung eines Gesamtbetriebsrats ist als „Muss-Vorschrift“ bei mehreren Betriebsräten in einem Unternehmen vorgesehen ( $\$ 47$ Abs. 1 BetrVG), ein Konzernbetriebsrat hingegen als „Kann-Bestimmung“ (§54 BetrVG). Ein Gesamtbetriebsrat ist zuständig für 
unternehmensübergreifende Themen ( $§ 50$ BetrVG). Die Aufgabenschwerpunkte liegen in informatorischen und koordinierenden Bereichen, er ist ,nicht übergeordnet und erst recht nicht weisungsbefugt “ (Fricke et al., 2018, S. 126), die Beteiligungsrechte verbleiben i. d. R. bei den einzelnen Gremien.

$\mathrm{Zu}$ den Aufgaben des Betriebsrats gehört es, vierteljährliche Betriebs- oder Abteilungsversammlungen zur Berichterstattung und zur Behandlung betrieblicher Themen durchzuführen (z. B. tarifpolitische oder wirtschaftliche Themen). Zur Darlegung der betrieblichen Lage, des Personal- und Sozialwesens, der Gleichstellung im Betrieb etc. wird der Arbeitgeber in eine jährliche Berichtspflicht genommen ( $§ 42-44$ BetrVG). In einem mindestens monatlichen Turnus sind Besprechungen des Betriebsrats (als vollständiges Gremium) und des Arbeitgebers vorgesehen ( $§ 74$ Abs. 1 BetrVG; Fricke et al., 2018, S. 171). Mit dem Betriebsrat getroffene Vereinbarungen auszuführen obliegt dem Arbeitgeber ( 77 Abs. 1 BetrVG).

\section{Betriebsvereinbarungen als schriftliches Ergebnis von Mitbestimmungsabläufen}

Das schriftliche Ergebnis von Mitbestimmungsabläufen wird als Betriebsvereinbarung bezeichnet (Fricke et al., 2018, S. 185). Beschlossene Betriebsvereinbarungen haben eine hohe Verbindlichkeit, sie gelten „unmittelbar und zwingend“ ( $\$ 77$ Abs. 4 Satz 1 BetrVG) so lange, bis sie gekündigt werden ( $§ 77$ Abs. 5 BetrVG), auslaufen oder ersetzt werden. Betriebsvereinbarungen können erzwingbar (bei bestehenden Mitbestimmungsrechten) oder freiwillig ( 88 BetrVG) abgeschlossen werden. Sie dürfen bestehende Arbeitnehmerrechte aus Gesetzen und Tarifverträgen nicht einschränken, nur erweitern. Arbeitsentgelte und tarifliche Themen sind inhaltlich i. d. R. ausgeschlossen ( $\$ 77$ Abs. 3 BetrVG). Eingesetzt werden sie somit für „die Regulierung von Sachverhalten, die nicht durch Tarifverträge geregelt sind“ (Nienhüser, 2005, S. 6). Häufig füllen sie „Branchentarifverträge aus, die entsprechende Öffnungsklauseln enthalten, etwa zur betrieblichen Umsetzung von tariflichen Arbeitszeitregelungen“ (ebd.).

\section{Die Rolle und Funktion der Einigungsstelle}

Entstehen Meinungsverschiedenheiten zwischen den Betriebsrat und Arbeitgeber, können Einigungsstellen involviert werden. Einigungsstellen nach dem BetrVG können entweder ständig (via Betriebsvereinbarung) oder punktuell, anlassbezogen errichtet werden ( $§ 76$ Abs. 1 BetrVG). Eine Einigungsstelle setzt sich personell aus einem neutralen Vorsitzenden und paritätisch benannten Beisitzer*innen der Konfliktparteien zusammen. Bei fehlendem Einvernehmen über die Besetzung oder darüber, ob es um ein Mitbestimmungsrecht geht, entscheidet 
das Arbeitsgericht ( $\$ 76$ Abs. 2 BetrVG). Eine genaue Anzahl der Beisitzer*innen wird nicht definiert. Betriebsräten wird empfohlen, für diese externe (honorarpflichtige) Fachleute zu benennen, um mit diesen fachlich zu überzeugen und über ein Druckmittel für potenziell kostspielige Einigungsstellenverfahren zu verfügen. Um die Stellen jeweils fachkompetent zu besetzen, haben sich in der Praxis bedarfsbezogene Errichtungen bewährt (Fricke et al., 2018, S. 176 ff.). Die Einigungsstelle entscheidet nach mündlicher Beratung nach dem Mehrheitsprinzip, zunächst ohne, im Bedarfsfall mit der Stimme des Vorsitzenden ( $§ 76$ Abs. 3 BetrVG). Sie hat ,unverzüglich tätig zu werden“ (ebd.), dem Vorsitzenden obliegt das genauere Ablaufprozedere. Für die Einigungsstelle wird zwischen einem erzwingbaren und einem freiwilligen Verfahren unterschieden. Ersteres wird auf Antrag des Betriebsrats oder Arbeitgebers initiiert und betrifft Mitbestimmungsangelegenheiten, der Schlichtungsspruch ist bindend. Arbeitsgerichtlich kann er lediglich als unwirksam erklärt werden. Das freiwillige Verfahren kann bei allen Meinungsverschiedenheiten angestoßen werden, die nicht mitbestimmungspflichtig sind. Es muss jedoch ein Einvernehmen beider Konfliktparteien zum Verfahren vorliegen und der Einigungsspruch hat nicht zwingend Verbindlichkeit (Fricke et al., 2018, S. 179 ff.).

\section{Rechte und Pflichten der Betriebsratsmitglieder}

Neben einem besonderen Kündigungsschutz ( $§ 103$ BetrVG) genießen Betriebsratsmitglieder weiteren Schutz: Sie „dürfen in der Ausübung ihrer Tätigkeit nicht gestört oder behindert“ (§ 78 BetrVG) werden und Bevorzugungen sind unzulässig. Dies wird - ebenso wie die Wahlbeeinflussung oder Tätigkeitsbehinderung des Betriebsrats bzw. der weiteren Gremien - strafrechtlich mit einer Freiheitsstrafe von bis zu einem Jahr bzw. mit Geldstrafen geahndet (§ 119 BetrVG). Zur Arbeitsbehinderung zählt auch die ,Zurückhaltung oder Verweigerung benötigter Informationen“ (Fricke et al., 2018, S. 190), Begünstigungen können z. B. durch übertriebene Auslagenerstattungen vorliegen (ebd.). Kommt der Arbeitgeber seiner Informationspflicht in bestimmten Bereichen nicht nach, liegt eine Ordnungswidrigkeit vor, die mit Geldstrafen sanktioniert werden kann ( $§ 121$ BetrVG). Für Betriebsratsmitglieder besteht eine Geheimhaltungspflicht für Betriebs- und Geschäftsangelegenheiten, die ,ausdrücklich als geheimhaltungsbedürftig bezeichnet“ ( $\$ 79$ Abs. 1 Satz 1 BetrVG) sind. Wird dagegen verstoßen, kann dies mit einer Freiheitsstrafe von bis zu einem Jahr bzw. monetär geahndet werden. Unter Umständen kann ein höheres Strafmaß vorliegen ( $§ 120$ BetrVG). Betriebsgeheimnisse müssen durch den Arbeitgeber jedoch explizit benannt werden und sind in der Praxis selten (Fricke et al., 2018, S. 304). 


\section{Aufgaben und Beteiligungsrechte des Betriebsrats}

$\mathrm{Zu}$ den allgemeinen Aufgaben des Betriebsrats gehört es zu überwachen, „dass die zugunsten der Arbeitnehmer geltenden Gesetze, Verordnungen, Unfallverhütungsvorschriften, Tarifverträge und Betriebsvereinbarungen durchgeführt werden“ (§ 80 Abs. 1 Nr. 1 BetrVG). Grundsätzlich hat er darauf zu achten, dass alle Betriebsangehörigen gleich, „rechtens und gerecht“ (Fricke et al., 2018, S. 174) behandelt werden (§ 75 Abs. 1 BetrVG). Geschlechtliche Gleichstellung, familiäre Vereinbarkeit, behinderungsspezifische Eingliederung, die Integration ausländischer und die Beschäftigung älterer Arbeitnehmer*innen sind explizit zu fördern ( $\$ 80$ Abs. 1 Nr. 2a, 2b, 4, 6-7 BetrVG). Zu den Aufgaben zählen u. a. ferner, nützliche Maßnahmen zu beantragen ( $§ 80$ Abs. 1 Nr. 2 BetrVG), Anregungen oder Beschwerden der Arbeitnehmer*innen entgegenzunehmen und zu bearbeiten ( $\S 80$ Abs. 1 Nr. 3 und 85 BetrVG), den betrieblichen Arbeits- und Umweltschutz zu fördern, mit den dafür zuständigen Stellen zusammenzuarbeiten und an Sitzungen zur Arbeitssicherheit teilzunehmen ( $\S 80$ Abs. 1 Nr. 9 und 89 BetrVG).

Um seine Aufgaben erledigen zu können, ist der Betriebsrat ohne Aufforderung ,rechtzeitig und umfassend vom Arbeitgeber zu unterrichten“ (§ 80 Abs. 2 Satz 1 BetrVG). Diese Bezeichnung wird als zu „Beginn einer Planung“ (Fricke et al., 2018, S. 201) ausgelegt. Als Informationsfluss vollzieht sich die Unterrichtung kontinuierlich und ist auf alle Betriebsratsmitglieder bezogen. „Maßstab sind ... die Informationen, die auch die Unternehmensleitung bekommt, um über eine geplante Maßnahme zu entscheiden.“ (ebd.) Der Betriebsrat kann weitere Unterlagen anfordern, (ausgewählte) sachkundige Arbeitnehmer oder Sachverständige hinzuziehen oder Gehaltslisten einsehen ( 80 Abs. 2 und Abs. 3 BetrVG).

Nach $\S 87$ Abs. 1 BetrVG hat der Betriebsrat in 13 Angelegenheiten Mitbestimmungsrechte. Diese betreffen u. a. die täglichen Arbeitszeiten, überwachende technische Einrichtungen, betriebliche Sozialeinrichtungen oder leistungsbezogene Entgelte. In diesen Bereichen sind Maßnahmen nur zulässig, wenn der Betriebsrat diesen zugestimmt hat oder eine ersetzende Entscheidung der Einigungsstelle vorliegt. Gleichfalls kann der Betriebsrat von sich aus

die Initiative zur Behandlung von Mitbestimmungsangelegenheiten ergreifen (Fricke et al., 2018, S. 212). Ein eingeschränktes Mitbestimmungsrecht besteht ferner bei „Änderungen der Arbeitsplätze, des Arbeitsablaufs oder der Arbeitsumgebung“ ( 991 BetrVG), die besonders belasten und im Kontrast zu arbeitswissenschaftlichen Erkenntnissen stehen. Spezielle Unterrichtungs- und Beratungsrechte ( $\S 90$ BetrVG) bestehen bei Bau- und weiteren künftigen Betriebsplanungen. Auch hier gilt der Grundsatz der rechtzeitigen Information mit allen 
notwendigen Unterlagen, d. h. bereits „,im Anfangsstadium der Planung“ (Fricke et al., 2018, S. 230). Für die Personalplanung ( $\$ 92$ BetrVG) besteht ein Unterrichtungs- und Beratungsrecht, auch wenn es keine geordnete Personalplanung gibt. Ergänzt wird dieser Bereich um explizite Vorschlagsmöglichkeiten zum Aufbau einer Personalplanung oder der Beschäftigungssicherung ( $§ 92 \mathrm{a}$ BetrVG) insgesamt (Fricke et al., 2018, S. 235). Weitere Befugnisse bestehen für interne Stellenausschreibungen ( 93 BetrVG), Personalfragebögen oder Beurteilungsgrundsätze ( $§ 94$ BetrVG), personelle Auswahlrichtlinien ( $\$ 95$ BetrVG) und für die Berufsbildung ( $\S 96-98$ BetrVG).

Im Bereich personeller Einzelmaßnahmen besteht ein Mitteilungs- und Widerspruchsrecht ( $§ 102$ BetrVG) bei Kündigungen. Auch über Neueinstellungen, Versetzungen etc. muss der Betriebsrat ab einer gewissen Unternehmensgröße informiert werden und kann mit gewissen Begründungen seine Zustimmung verweigern, die lediglich arbeitsgerichtlich ersetzt werden kann (§ 99 BetrVG). Hiervon ausgenommen sind leitende Angestellte ( 105 BetrVG).

$\mathrm{Ab}$ einer Größe von über 100 Arbeitnehmer*innen muss in einem Unternehmen ein dreibis siebenköpfiger Wirtschaftsausschuss gebildet werden, der monatlich tagt und wirtschaftliche Angelegenheiten wie z. B. die monetäre Unternehmens- bzw. Absatzlage oder Rationalisierungsmaßnahmen berät und die Informationen an den Betriebsrat weitergibt. Mindestens ein Ausschussmitglied muss dem Betriebsrat angehören ( $§ 106-108$ BetrVG). Anstehende, für die Beschäftigten potenziell nachteilige Betriebsänderungen wie z. B. örtliche Verlegungen muss der Arbeitgeber mit dem Betriebsrat ab einer Wählerschaft von 20 Personen beraten ( 111 BetrVG). Über einen Interessenausgleich bzw. einen Sozialplan bei bestehenden Nachteilen ist nach $\S 112$ BetrVG zu verhandeln.

\section{Position des Betriebsrats nach dem BetrVG}

Formal gesehen enthält das BetrVG abgestufte Rechte für das betriebliche Arbeitnehmergremium, die von Informations- bzw. Unterrichtungs- über eingeschränkte bzw. zwingende Beteiligungs- bis zu Mitbestimmungsrechten reichen. Thematisch-inhaltlich macht sich eine Steigerung bemerkbar: Die Rechte des „Betriebsrats sind umso größer, je weiter sie von den strategischen Unternehmensentscheidungen ... entfernt sind“ (Kißler et al., 2011, S. 67). Als defizitär und nicht weitreichend genug werden insbesondere die eingeschränkten Befugnisse im wirtschaftlichen Bereich sowie im Bereich technischer Neuerungen kritisiert (ebd., S. $66 \mathrm{f}$.).

Im Hinblick auf die rechtliche Anlage ist das Verhältnis des Betriebsrats innerhalb des betrieblichen Beziehungsgefüges zwischen Management und Belegschaft ein 
spannungsreicher „Drahtseilakt“ (Minssen \& Riese, 2007, S. 9), was auch in der Kennzeichnung als „Konfliktpartnerschaft“ (Müller-Jentsch, 1999, S. 9) zum Ausdruck kommt. Die Bezeichnung als „Sozialpartnerschaft“ (Greifenstein \& Kißler, 2010, S. 15) betont demgegenüber die Auslegung einer friedenssichernden, integrativen und ausgewogenen Position des Betriebsrats, wie sie mit der vertrauensvollen Zusammenarbeit nach $\S 2$ Abs. 1 BetrVG angelegt ist. Sie wird in Abschnitt 2.2.3 genauer betrachtet.

\section{Merkmale des deutschen Modells}

Die mit dem BetrVG gegebene Struktur der Betriebsratsarbeit wird als deutsches Modell der industriellen Beziehungen bezeichnet. Dieses Modell umfasst nach Müller-Jentsch (1995, S. 13 ff.) fünf wesentliche Aspekte:

- Erstens die Dualität der Gestaltung. Gemeint ist die zweigleisige Struktur der betrieblichen und gewerkschaftlichen Interessenvertretung mit ihren unterschiedlichen Handlungslogiken (siehe Abschnitt 2.2.3).

- Zweitens den Betriebsrat als „,intermediäre Institution“ (ebd., 1999, S. 9), dem rechtlich definiert nicht nur die Interessenvertretung der Arbeitnehmer*innen obliegt, sondern der auch einer Wirtschaftslogik verpflichtet ist. Gedeutet wird dies als Zusammenführung der gegensätzlichen Interessenlagen von Kapital und Arbeit in einer Instanz. Diese Intermediarität ist per se nicht für alle Arbeitnehmervertretungen zutreffend, sondern gilt als charakteristisch deutscher Weg, gleichfalls bei den Gewerkschaften.

- Drittens die hohe staatliche Regelungsdichte (Verrechtlichung), die in Form des Tarifvertragsgesetzes, des BetrVG und weiterer Rechtsnormen zum Ausdruck kommt und mit der der Gesetzgeber „als ,dritte Partei‘ die Bühne der industriellen Beziehungen betritt“ (ebd., 1995, S. 14).

- Viertens die Zentralisierung der Organisationsstrukturen der Arbeitnehmer- und Arbeitgeberverbände sowie die zentral geltenden Tarifverträge. Diese wird jedoch angesichts vermehrter Öffnungsklauseln und anderer Entwicklungen mittlerweile angezweifelt (Jakobi, 2007, S. 25).

- Fünftens die stellvertretende Funktion der Interessenvertretungen (Repräsentativität) mit ihrer Wahllegitimierung und davon unabhängigen Handlungsbefugnissen ohne direkte Zustimmungsnotwendigkeit.

Bei diesen Merkmalen des deutschen Modells der Arbeitsbeziehungen muss ergänzt werden, dass in der hiesigen Privatwirtschaft oftmals keine betriebliche Interessenvertretung besteht 
oder keine Tarifbindung gegeben ist. Nach den aktuellen Daten des Betriebspanels des Instituts für Arbeitsmarkt- und Berufsforschung der Bundesagentur für Arbeit (IAB-Betriebspanel) 2018 gibt es in ca. 9 \% der privatwirtschaftlichen Betriebe (ab fünf Beschäftigten, ohne Landwirtschaft) einen Betriebsrat, mit einer deutlichen Zunahme der Gremien bei den großen Betrieben. ${ }^{12}$ Bezogen auf die Beschäftigtenzahlen arbeiteten im privatwirtschaftlichen Bereich im Jahr $201842 \%$ der Beschäftigten in West- und 35 \% der Beschäftigten in Ostdeutschland in Betrieben mit einem Betriebsrat. Die Anzahl der Betriebe ohne Tarifbindung wird für dasselbe Jahr mit $25 \%$ beziffert. Wiederum bezogen auf die Beschäftigtenzahlen haben $46 \%$ der Beschäftigten in der Privatwirtschaft in einem Betrieb mit Branchentarifbindung gearbeitet (Ellguth \& Kohaut, 2019, S. 291 ff.).

$\mathrm{Zu}$ beachten ist zudem, dass sich Beschreibungen des deutschen Modells der Arbeitsbeziehungen i. d. R. auf den privatwirtschaftlichen Sektor fokussieren und der öffentliche Dienst und der kirchliche Bereich unberücksichtigt bleiben. Im öffentlichen Dienst richtet sich die Mitbestimmung nach den Personalvertretungsgesetzen des Bundes und der Länder. Gegenüber der Privatwirtschaft bestehen anders gelagerte Arbeitsbeziehungen und eingeschränkte Beteiligungsrechte. Problematisiert werden u. a. die unterschiedlichen Vorgaben in den Ländern und für den Tarif- und Beamtenbereich, die eine weitere gewerkschaftliche Organisation und die Rechtsprechung erschweren (Brehmer, 2014, S. 14 ff.). Auf eine weitere Darstellung der Beteiligungsvorgaben im öffentlichen Sektor wird im Rahmen dieser Arbeit verzichtet, da kommunale/öffentliche Träger von WfbM wenig verbreitet sind (siehe etwa Schlummer, 2004, S. 63). Die Vorgaben des kirchlichen Bereichs sind hingegen für WfbM sehr relevant, da sich ein größerer Teil der Werkstätten in kirchlicher Trägerschaft befindet: Im Jahr 2019 ordneten sich knapp $40 \%$ der Mitglieder der BAG WfbM den kirchlichen Trägerverbänden zu (BAG WfbM, 2019). Im Folgenden werden deswegen die Beteiligungsgremien in diesem Sektor gesondert betrachtet.

\subsubsection{Der „dritte Weg“ der Kirchen}

Aufgrund des kirchlichen Selbstbestimmungsrechts nach Art. 140 GG bestehen in Deutschland für die verfassten Kirchen und deren Organisationen eigene arbeitsrechtliche Grundlagen,

\footnotetext{
12 Das IAB-Betriebspanel ist eine seit 1993 jährliche Wiederholungsbefragung von derzeit ca. 16.000 Betrieben (Zufallsziehung auf Basis aller bei der BA gemeldeten Betriebe mit sozialversicherungspflichtigen Beschäftigten). Nähere Informationen sind verfügbar unter: https://www.iab.de/de/erhebungen/iab-betriebspanel/informationen-zum-panel.aspx
} 
die auch die Interessenvertretung der Beschäftigten in den kirchennahen Einrichtungen der Diakonie (der Diakonischen Werke der Evangelischen Kirche) und der Caritas (der Caritasverbände der Diözesen) betreffen. In Anlehnung an Jakobi (2007, S. 17 f.) lässt sich dazu die zusammenfassende Bezeichnung konfessioneller Bereich verwenden, der die heterogene Struktur des Sektors betont. Anstelle eines Betriebsrats müssen in dem konfessionellen Bereich Gremien der Mitarbeitervertretung (MAV) eingerichtet werden. Für die katholische Kirche und deren Dienststellen richtet sich die Einrichtung i. d. R. nach der „Rahmenordnung für eine Mitarbeitervertretungsordnung “13 (MAVO) aus, wobei verschiedene Abwandlungen in den Diözesen bestehen. Für die Dienststellen und Einrichtungen der Diakonie besteht das „Kirchengesetz über Mitarbeitervertretungen in der Evangelischen Kirche in Deutschland“ (MVG-EKD). ${ }^{14}$

Anders als im privatwirtschaftlichen Bereich ist die Bildung einer MAV in den kirchlichen Einrichtungen nicht dem potenziellen Engagement der Arbeitnehmer*innen oder Arbeitgeber überlassen, sondern ab fünf wahlberechtigten Mitarbeitenden verbindlich vorgesehen. Die Gründung einer MAV ist eine Pflichterfüllung der Dienststellenleitung ( $\$ 7$ Abs. 1 MVGEKD) bzw. des Dienstgebers ( $(10$ Abs. 1 MAVO). Wesentlich ist hierbei das Verständnis als Dienstgemeinschaft, was in den Bezeichnungen als Dienstgeber und Dienstnehmer*in zum Ausdruck kommt. Die Dienstgemeinschaft wird in der Präambel Satz 3 MVG-EKD als ,gemeinsame Verantwortung für den Dienst der Kirche und ihrer Diakonie“ beschrieben und in ähnlicher Art auch für die katholische Kirche bestimmt (Präambel Satz 4 MAVO). Als Teil des kirchlichen Dienstes verbindet die Dienstgemeinschaft Dienstgeber und -nehmer*innen unabhängig von der beruflichen Stellung und verpflichtet diese zur Zusammenarbeit (siehe hierzu Ensinger, 2006, S. 61 ff.). Nach der Auffassung einer einheitlichen Dienstgemeinschaft werden die Interessengegensätze von Kapital und Arbeit für den konfessionellen Bereich tendenziell negiert, wie dies mit dem Ausschluss von Streikrechten als Mittel des Arbeitskampfes (Richardi, 2020, S. 124 ff.) zum Ausdruck kommt. Der Begriff der Dienstgemeinschaft bleibt insgesamt „schillernd und vage“ (Jakobi, 2007, S. 66) und vermag die unterschiedlichen Interessenlagen von Arbeitnehmer*innen und Arbeitgeber letztlich nicht vollends so aufzulösen, wie dies mit der Gemeinschaftsverpflichtung angelegt ist (Ensinger, 2006, S. 63).

13 In der Fassung des einstimmigen Beschlusses der Vollversammlung des Verbandes der Diözesen Deutschlands vom 20. November 1995, zuletzt geändert am 19.06.2017.

${ }^{14}$ Neufassung vom 1. Januar 2019 (ABl. EKD S. 2), zuletzt geändert am 13. November 2019 (AB1. EKD S. 322). 
Die Gremienstrukturen der MAV und die formalen Abläufe ähneln derjenigen des Betriebsrats. Die regelhafte Amtszeit beträgt vier Jahre (§ 13 Abs. 2 S. 2 MAVO; $§ 15$ Abs. 1 MVG-EKD), es ist eine vorsitzende Person zu wählen (§ 14 Abs. 1 MAVO; § 23 MVG-EKD), eine jährliche Mitarbeiterversammlung durchzuführen etc.

Für die Mitarbeitergremien der katholischen Kirche werden in der MAVO verschiedene Informations- ( $\S 27,27 \mathrm{a})$ und Beteiligungsrechte $(\S 28)$ benannt, die hinsichtlich ihrer Reichweite unterschieden werden. Möglich sind eine Anhörung und Mitberatung (§§ 29-31 MAVO), initiative Vorschlags- (§ $32 \mathrm{MAVO}$ ) und Antragsrechte ( $37 \mathrm{MAVO}$ ) sowie Zustimmungsrechte in verschiedenen Angelegenheiten (§33 MAVO). Die inhaltlichen Bereiche, auf die sich die Beteiligungsrechte beziehen, sind vorgegeben und entsprechen sich teilweise. So besteht etwa bei der täglichen Arbeitszeit ein Mitberatungs-, ein Vorschlags-, ein Antragsund (bezogen auf die Dienststelle) ein Zustimmungsrecht. Im Anwendungsbezug muss beachtet werden, ob eine Angelegenheit vonseiten der MAV oder des Dienstgebers eingebracht wird, wonach sich das weitere Prozedere und die Befugnisse ausrichten (Richardi, 2020, S. 261). Zur Klärung von Streitigkeiten der MAV sind in den Diözesen Einigungsstellen gebildet (§ 40 Abs. 1 MAVO). Diesen übergeordnet kann das kirchliche Arbeitsgericht konsultiert werden ( $\$ 47$ Abs. 4 MAVO).

Das MVG-EKD beinhaltet bezogen auf die Beteiligungsrechte eine andere Gliederung. Benannt werden Fälle der Mitbestimmung (§ 39-40), der eingeschränkten Mitbestimmung (§ 41-43), der Mitberatung ( $(45-46)$ sowie Initiativ- und Beschwerderechte (§ 47-48). Beteiligungstatbestände sind personelle, soziale und organisatorische Angelegenheiten. Bei Unstimmigkeiten in sozialen und organisatorischen Angelegenheiten können seit Anfang 2020 Einigungsstellen anlassbezogen oder als dauerhafte Instanz gebildet werden (§ 36a MVGEKD), die ersetzende Entscheidungen treffen. Daneben ist bei Streitigkeiten der Weg über die Kirchengerichte sowie über den Kirchgengerichtshof der Evangelischen Kirche ( $§ 60$ MVGEKD) eröffnet.

Im Vergleich zum BetrVG bestehen im evangelischen und katholischen Mitarbeitervertretungsrecht einige Unterschiede. Der Kündigungsschutz der MAVO gilt bspw. als weniger umfassend (Richardi, 2020, S. 252), die Anzahl der Mitarbeiterversammlungen ( $\$ 31$ MVGEKD) ist reduziert, der Wirtschaftsausschuss wurde erst 2017 als fakultative Einrichtung eingeführt (Richardi, 2020, S. 255) und „Fragen der betrieblichen Lohngestaltung“ (§ 87 Abs. 1 Nr. 10 BetrVG) sind im kirchlichen Mitarbeitervertretungsrecht keine Mitbestimmungsangelegenheiten (Richardi, 2020, S. 279). Insgesamt gelten die Mitbestimmungsrechte als eingeschränkt (Deppisch, Jung \& Schleitzer, 2014, S. 124). Bemängelt wird insbesondere das 
Rechtsschutzverfahren über die Kirchengerichte als „Rechtsschutz zweiter Klasse“ (ebd., S. 154). Abzuwarten bleibt, wie sich die Situation mit den neu gebildeten Einigungsstellen im evangelischen Mitarbeitervertretungsrecht entwickelt.

Nach der Skizzierung dieser rechtlichen Grundlagen der Arbeitnehmergremien in der Privatwirtschaft und im konfessionellen Bereich wird nun herausgearbeitet, wie sich deren Sinn und Zweck in gesellschaftlichen Zusammenhängen deuten lässt.

\subsubsection{Demokratietheoretische Bezüge und funktionelle Deutungen}

Je nach dem disziplinären oder interessengeleiteten Hintergrund werden in den Betrachtungsweisen betrieblicher Mitbestimmung unterschiedliche Aspekte betont, bspw. der betriebswirtschaftliche Nutzen oder Nachteil partizipativer Prozesse in ökonomisch orientierten Analysen (Michaelis \& Picot, 1987), die Einbindung in Innovationsprozesse in zukunftsorientierten Betrachtungen (Kriegesmann, Kley \& Kublik, 2010) oder betriebliche Abhängigkeits- und Herrschaftsbeziehungen in industriesoziologischen Abhandlungen (Kotthoff, 1981). Vorhandene Berichterstattungen bewegen sich zwischen „Tradition und Effizienz“ (Streeck \& Kluge, 1999) der Arbeitnehmervertretungen, die durch Veränderungen der Arbeitswelt vor anhaltenden Herausforderungen stehen, mit denen ein Bedeutungsverlust assoziiert wird.

Kennzeichnend für die Theorie der Mitbestimmung sind dementsprechend unterschiedliche Lesarten, die auf unterschiedlichen Demokratievorstellungen oder wirtschaftsdemokratischen Überlegungen beruhen. Im Kanon der verschiedenen Deutungsweisen lassen sich mit Kißler et al. (2011, S. 26 ff.) drei differierende Ideen herausarbeiten, mit denen betriebliche Mitbestimmung assoziiert wird.

1. Mitbestimmung als formal-analytische oder konservativ-demokratische Einrichtung

Diese Sichtweise sieht Demokratie durch Institutionen formalisiert und durch Wahlakte legitimiert. Sie folgt einer Steuerungsperspektive und der „Konkurrenzdemokratie“ im Sinne Fraenkels. In diesem neopluralistischen Ansatz geht es um die „Legitimierung von Vielfalt in der politischen Willensbildung“ (Massing, 2012, S. 261) durch die Einflussnahme von (sozialen) Interessenvertretungen. In seinen Werken folgt Fraenkel der Annahme, dass der Mensch bei seinem politischen Handeln von als Konsens geteilten gesellschaftlichen Werten geprägt sei, er aber dennoch immer auch durch Eigeninteressen motiviert werde. Dies betrachtet Fraenkel als legitim und sieht es als wesentliches Element westlicher Demokratien an, dass Bürger*innen ihre partikularen Interessen frei und ungehindert in kollektiven Interessenvertretungen 
organisieren (Fraenkel, 2007, S. 291 ff.; Massing, 2012, S. 261 ff.). In dieser Auslegung ist die Mitbestimmungsidee grundlegend positiv konnotiert, ein normativer Beteiligungsanspruch bleibt jedoch verkürzt und auf bestimmte Verfahrenswege bzw. den politischen Bereich beschränkt, ohne weitergehende wirtschaftsdemokratische Einflüsse. Praktisch gesehen beschreiben Kißler et al. (2011, S. 27) diese Deutung als „Pseudopartizipation“, wonach Beteiligungsaktivitäten ohne entscheidenden Einfluss ausgeübt würden.

\section{Mitbestimmung als Managementstrategie}

Sowohl formalisierte Beteiligungsformen als auch offene partizipative Verfahren moderner Organisationsführung (Hucker, 2008) können wirtschaftlich-unternehmerische Ziele verfolgen. Als Managementstrategien zielen offene partizipative Verfahren bspw. darauf, eine Akzeptanz, das Erfahrungswissen oder die Motivation der Beschäftigten aufzugreifen und zu steigern. Zwar sind damit auch partizipative Lern- und Qualifizierungsmöglichkeiten verbunden, betont wird jedoch eher die unternehmens-/herrschaftsstabilisierende Komponente und die offene Frage danach, inwieweit Beschäftigungsinteressen mit solchen Verfahren durchgesetzt werden können (Kißler et al., 2011, S. 28 ff.). So beziehen sich managementorientierte Beteiligungsangebote inhaltlich tendenziell eher auf tätigkeitsbezogene Themen als auf Personalangelegenheiten oder andere Bereiche, die einen Ausgleich unterschiedlicher Interessenlagen fokussieren (Hucker, 2008, S. 297 f.).

\section{Mitbestimmung als Partizipationsverfahren}

Im Sinne einer komplexen Demokratievorstellung nach Scharpf (1973) bezieht sich Mitbestimmung auf unmittelbare Formen in allen gesellschaftlichen Bereichen, z. B. auch in der Wirtschaftsordnung. Es geht nicht nur um Herrschaftslegitimierung, sondern um gestalterische Lernprozesse und die Möglichkeit, diese mit bürgerlichen Rechten und Pflichten einzunehmen (Kißler et al., 2011, S. 32 ff.). Vorausgesetzt wird die Überwindung politisch apathischer Haltungen, wobei apathische Haltungen durch die Komplexität politischer Entscheidungen und den Wettbewerb mit anderen „Individualinteressen“ (Scharpf, 1973, S. 119) als notgedrungen gegeben gedeutet werden. Ein Partizipationswille kann folglich oftmals nur im Kleinen erweckt werden, d. h. mit spezialisierter und gegenwärtiger Einflussnahme in greifbaren Bereichen (ebd., S. 118 ff.).

In diesem Denkmodell ist Mitbestimmung als institutionalisierte Form auf allen wirtschaftlichen Ebenen und auf einer komplementären, individuellen Ebene der Subjektbildung 
notwendig. Emanzipatorische Selbstbestimmung wird dabei zum Postulat erhoben und eine überspitzte Polarisierung mit formalisierter, integrativer Mitbestimmung ad absurdum geführt. Indem Mitbestimmung als „Durchgangsstadium“ (Kißler et al., 2011, S. 37) und Mittel einer stetig zunehmenden Selbstbestimmung von immer mehr gesellschaftlichen Kreisen begriffen wird, übersteigt sie formal-analytische Theorien, behält einen normativen Bezugsrahmen und kann als „,(Belegschafts-)Partizipation“ (ebd., S. 38) im Einklang mit Selbstbestimmung stehen.

\section{Funktionelle Deutungen}

Neben verschiedenen Auslegungen finden sich zur betrieblichen Mitbestimmung unterschiedliche funktionelle Deutungen, die dieser zugesprochen werden. Grundlegend sind z. B. die der Friedensstiftung und Demokratisierung. Für das deutsche System gilt, dass betriebliche Beteiligung kooperativ als „Friedensarena“ (Kißler et al., 2011, S. 48) angelegt ist (siehe Abschnitt 2.2.1), während Verhandlungen im Sinne der Tarifautonomie konflikthaft (mit Arbeitskampfmitteln) ausgetragen werden können. Ausgehend von dieser Struktur werden betrieblicher Mitbestimmung integrative, innovative und unternehmenskulturelle Funktionen zugeschrieben.

Die neokorporatistische oder integrative Lesart betont, dass sich mit der Zuweisung von Mitbestimmung eine Integration in das vorhandene Beziehungssystem vollzieht. Potenzieller Zündstoff wird in anerkannte Verfahrenswege kanalisiert und eine Mitverantwortung an dem Geschehen eingeführt (Kißler et al., 2011, S. 51). Für den Betriebsrat wird dieses Verhältnis in einer kooperativen Deutung als „Sozialpartnerschaft“ (Greifenstein \& Kißler, 2010, S. 15) charakterisiert. Es kommt zu einer organisierten und verbindlichen Eingliederung der Interessenlagen in Entscheidungsprozesse, im politikwissenschaftlichen Terminus als Neo- oder liberaler Korporatismus bezeichnet (Nohlen, 2002, S. 449).

Andere Lesarten sehen Mitbestimmung eher mit innovativen Aufgaben und einer unternehmenskulturellen Bindung bezweckt. Wie bereits in der Deutung als Managementstrategie angesprochen, kann Mitbestimmung Lern- und Kommunikationseffekte erzeugen und Innovationen voranbringen. Zum einen kann über die Vertretungsorgane Organisationswissen erschlossen werden, zum anderen können Neuerungen entsprechend kommuniziert und nachvollzogen werden, was wiederum mit einer höheren Akzeptanz verbunden wird. Dieses Potenzial gilt als fruchtbar für Organisationen mit flachen Hierarchien (Greifenstein \& Kißler, 2010, S. 18). 
Mit einem normativen Anspruch wird Mitbestimmung als Kennzeichen einer guten Unternehmenskultur ausgegeben, wie dies aktuell vermehrt unter „Corporate Social Responsibility“ praktiziert wird. Hiernach werden soziale, gesellschaftliche und ökologische Ansprüche freiwillig in der Unternehmensführung beachtet und umgesetzt, was die Attraktivität als Arbeitgeber und eine Identifikation mit diesem steigern soll (Dubielzig \& Schaltegger, 2005). $\mathrm{Zu}$ beachten ist nach Kißler et al. (2011, S. 55), dass sich diese (Unternehmens-)Kulturfunktion mit personalpolitischen und auf Konsens ausgerichteten Strategien verbinden kann und Mitbestimmung somit tendenziell lediglich eine dienliche Stellung einnimmt.

Inwieweit die beschriebenen Funktionen der Mitbestimmung künftig weiter ausgefüllt werden können, bleibt angesichts wandelnder Arbeitsmarktsektoren, veränderter Arbeitsverhältnisse, konkurrierender partizipativer Managementkonzepte und weiterer gesellschaftlicher Veränderungen offen (Hucker, 2008; Kißler et al., 2011, S. 56 f.). So verschieben bspw. tarifrechtliche Öffnungsklauseln (konflikthafte) Aushandlungen auf die betriebliche Ebene, was der friedensstiftenden Funktion entgegenläuft. Oder der Integrationsfunktion entschwindet anlässlich des wachsenden mitbestimmungsschwachen Dienstleistungsbereichs (Ellguth \& Kohaut, 2019) und der schrumpfenden Montanindustrie - die betriebsrätliche und gewerkschaftliche Basis (Greifenstein \& Kißler, 2010, S. 17 f.). Unabhängig von den Funktionszuschreibungen, die mit betrieblicher Mitbestimmung verbunden werden, lässt sich fragen, ob es sich bei den gegebenen Strukturen um Partizipation handelt. Im Folgenden wird eine Deutungsperspektive vorgestellt, die genau diese Frage analysiert.

\subsubsection{Partizipation nach Kißler, Greifenstein und Schneider}

Um einen Analyserahmen für die verschiedenen Beteiligungsverfahren im Bereich von Arbeit und Beschäftigung zu schaffen, skizzieren Kißler, Greifenstein und Schneider (2011, S. 40) in ihrer Monografie „Die Mitbestimmung in der Bundesrepublik Deutschland“ ein demokratisches Partizipationsverständnis. Hierzu bestimmen die Autoren Partizipation als „Beteiligung an Entscheidungsprozessen, zu dem Zweck, dort die eigenen Interessen oder die Interessen von Wählerinnen und Wählern durchzusetzen“ (ebd.). Als demokratische Partizipation zeichne sich diese aus, sofern gewisse Voraussetzungen gegeben sind.

Zunächst sieht ihre Definition von Partizipation eine Bestimmung anhand von drei Anzeichen vor (ebd., S. 41): Erstens ist Partizipation von Interessen begleitetes soziales Handeln. Zweitens erfolgt ein direkter oder delegierter, indirekter Einbezug bei Entscheidungsfindungen. Drittens ist dieser mit Befugnissen im Sinne einer Machtbegrenzung verbunden. Zur 
demokratischen Partizipation erhöht sich diese Beteiligung, wenn sie den drei Grundsätzen der Gleichheit, Freiheit und Verbindlichkeit folgt. Das heißt, im Bereich von Arbeit und Beschäftigung muss der Einbezug prinzipiell in aktiver und passiver Verfahrensweise allen Beschäftigten offenstehen. Partizipation kann nicht erzwungen werden und muss mit ernst zu nehmenden Entscheidungsbefugnissen verbunden sein, die reine Rede- oder andere Scheinverfahren übersteigen.

Als Weiterführung dieser demokratischen Prinzipien halten Kißler et al. (2011) drei Bestimmungsmerkmale zur qualitativen Beurteilung partizipativer Vorgänge fest: die „Regelproduktion“, die „Partizipationsquote“ und den „Partizipationsgrad“ (S. 41). Überprüfen lassen sich diese in Form der folgenden Fragen (ebd.):

1. Woraus geht das Verfahren hervor?

2. Wer hat das Recht, am Verfahren teilzunehmen?

3. Beeinflusst das Verfahren Entscheidungen?

Mit diesen Maßstäben kann eine Qualitätsprüfung von Beteiligungsangeboten erfolgen. Bezogen auf die potenziell gegebenen Wege der deutschen Mitbestimmung ergibt sich nach Kißler et al. ein ernüchterndes Bild. Für die delegierten Beteiligungsverfahren auf den Ebenen des Betriebs (Betriebsrat) und des Unternehmens (Aufsichtsrat) liegt zwar eine Regelproduktion in Form der gesetzlichen Normierungen vor, bezogen auf die zwei anderen Merkmale zeigen sich jedoch Abschläge der demokratischen Qualität. Die Partizipationsquote bleibt auf gewählte Personen reduziert. Der Partizipationsgrad ist - anlässlich eingeschränkter Rechte des Betriebsrats (etwa in wirtschaftlichen Angelegenheiten) und einer lediglich in der Montanindustrie bestehenden egalitären Zusammensetzung des Aufsichtsrats - insgesamt schwach (ebd., S. 42 f.).

Mit diesem Partizipationsverständnis aus der betrieblichen Mitbestimmungstheorie liegt ein knappes Konzept vor, das sich zur Abgrenzung von undemokratischen Beteiligungsverfahren und für qualitative Beurteilungen eignet. Unterschiede zu dem erkenntnistheoretischen Ansatz von Scheu und Autrata (2013) liegen u. a. darin, dass hier die Einflussnahme auf Entscheidungen und der Machtbegriff stärker fokussiert werden, was dem Kontext der industriellen Beziehungen und dem dort gängigen Duktus der potenziell konträren Kapital- und Belegschaftsinteressen entspricht. Gleichfalls gilt Partizipation nach Kißler et al. als übertragbar, eine Interessenausrichtung kann delegiert (und nicht nur subjektiv bestimmbar) erfolgen. 


\subsection{Zwischenfazit}

Zusammenfassend zeigt sich, dass Partizipation ein schillernder Begriff ist, für den unterschiedliche, nicht immer explizit benannte Lesarten bestehen. So ist Partizipation im derzeitigen Fachdiskurs zu Behinderungen noch kein eigenständiger, spezifizierter Bezugsrahmen. Partizipation nimmt hier vielmehr die Position eines eher unscheinbaren Beiwerks des Teilhabebegriffs ein. Die näher vorgestellten Definitionen, die aus anderen Fachdebatten stammen, betonen unterschiedliche Aspekte. Bei Scheu und Autrata (2013, S. 268 ff.) steht der Subjektbezug im Vordergrund. Wesentlich ist bei ihnen, dass sich der Gegenstand und die Zielrichtung von Partizipation (Erweiterung von individueller Lebensqualität vs. solidarischer Einflussnahme auf größere gesellschaftliche Bezüge) nur durch subjektive Sinnsetzungen ergeben können. Der vorgestellte Ansatz aus der betrieblichen Mitbestimmungsforschung begreift Partizipation demgegenüber prinzipiell als delegierbar und betont demokratische Qualitäten. Die Quintessenz von Stufenmodellen verweist wiederum darauf, dass Partizipation unterschiedliche Formen (Stufen) annehmen kann und in der (politischen) Praxis viele Scheinverfahren kursieren.

Bei aller Unterschiedlichkeit stimmen die Ansätze dahingehend überein, dass es bei Partizipation um einflussnehmende Prozesse geht (so auch de Nève \& Olteanu, 2012, S. 13), um eine Machtteilhabe am Geschehen. Dies wird mitunter als „Selbstwirksamkeitserfahrungen“ oder „Empowermentprozesse“ (Windisch, Rohrmann \& Düber, 2015, S. 398) deklariert. Diese Machtteilhabe ist auf Egalität gerichtet und kann sich in politischen, sozialen, betrieblichen, leistungserbringenden oder anderen Kontexten vollziehen. Die betrachteten Partizipationsverständnisse ähneln sich darin, dass es bei Partizipation um eine Einflussnahme geht, die mit einer kategorischen Umverteilung von Macht verbunden ist. Das heißt, eine Einflussnahme ist im Verhältnis der Beteiligten ausnahmslos angelegt, sie kann sich tatsächlich vollziehen und ist nicht an Bedingungen geknüpft. Es kristallisiert sich somit eine auf Egalität gerichtete Einflussnahme als Wesenskern von Partizipation heraus: Als Arbeitsdefinition kann festgehalten werden, dass Partizipation ein aktives, prozesshaftes, an demokratischen Qualitäten orientiertes, einflussnehmendes und machtvolles Handeln ist.

Mit dieser Begriffsauslegung von Partizipation wird im Folgenden das durch die WMVO initiierte Beteiligungsgeschehen in WfbM in Verbindung gebracht und das durch Werkstatträte ausgeübte Handeln betrachtet. Zur genaueren Erörterung von demokratischen Qualitäten bieten sich wiederum die Maßstäbe zur Musterung für Partizipationsverfahren von Kißler et al. (2011, S. 41) an. Das heißt, es gilt den Blick darauf zu richten, wie ein Verfahren 
entstanden ist, wer alles beteiligt wird und wie weitreichend Einfluss genommen werden kann. Ein weitergehendes, subjektives Partizipationsverständnis, wie es von Scheu und Autrata vorliegt, ist für die vorliegende Arbeit weniger geeignet, da nicht subjektive Erfahrungsprozesse, sondern strukturelle Umsetzungsprozesse im Fokus stehen.

Neben der Begriffsklärung von Partizipation wurden in diesem Kapitel Betriebsräte in der Privatwirtschaft und Mitarbeitervertretungen im kirchlichen Bereich als primäre Beteiligungsform im Bereich von Arbeit und Beschäftigung betrachtet. Zudem wurden Deutungsweisen der betrieblichen Mitbestimmung und verschiedene Funktionszuschreibungen vorgestellt. Darauf aufbauend wird nun auf die in der Einleitung der Arbeit aufgeworfenen Fragen eingegangen, warum überhaupt Werkstatträte bestehen und wie sich die Arbeitsbeziehungen in WfbM charakterisieren lassen. 